\title{
Designing Vibrotactile Widgets with Printed Actuators and Sensors
}

\author{
Christian Frisson ${ }^{1}$, Julien Decaudin ${ }^{1}$, Thomas Pietrzak ${ }^{2}$, Alexander Ng $^{3}$, \\ Pauline Poncet $^{4}$, Fabrice Casset ${ }^{4}$, Antoine Latour ${ }^{5}$, Stephen A. Brewster ${ }^{3}$ \\ ${ }^{1}$ Inria Lille, ${ }^{2}$ Université Lille $1,{ }^{3}$ University of Glasgow, ${ }^{4}$ CEA LETI, ${ }^{5}$ CEA LITEN \\ first.last @ $\left\{{ }^{1}\right.$ inria.fr ; ${ }^{2}$ univ-lille1.fr ; ${ }^{3}$ glasgow.ac.uk $;{ }^{4,5}$ cea.fr $\}$
}

\begin{abstract}
Physical controls are fabricated through complicated assembly of parts requiring expensive machinery and are prone to mechanical wear. One solution is to embed controls directly in interactive surfaces, but the proprioceptive part of gestural interaction that makes physical controls discoverable and usable solely by hand gestures is lost and has to be compensated, by vibrotactile feedback for instance. Vibrotactile actuators face the same aforementioned issues as for physical controls. We propose printed vibrotactile actuators and sensors. They are printed on plastic sheets, with piezoelectric ink for actuation, and with silver ink for conductive elements, such as wires and capacitive sensors. These printed actuators and sensors make it possible to design vibrotactile widgets on curved surfaces, without complicated mechanical assembly.
\end{abstract}

\section{ACM Classification Keywords}

H.5.2. Information Interfaces and Presentation (e.g. HCI): Haptic I/O

\section{Author Keywords}

Vibrotactile feedback; printed electronics; printed actuators; printed sensors; widgets; piezoelectric ink; thin-film actuators

\section{INTRODUCTION}

Computer peripherals, remote controls and dashboards all use physical buttons and sliders for input. The physicality of these controls provide essential usability benefits, such as help to locate the controls for eyes-free interaction or immediate feedback. The drawback is that these controls require complex assembly, are expensive to manufacture, and are prone to mechanical wear.

The solution we have today is to replace physical controls by multitouch sensors. It alleviates the issues mentioned earlier, however it also loses the advantages. A common solution is to use vibrations to replace the sensations of physical controls [3]. Tactile feedback coupled with pointing input is sufficient to

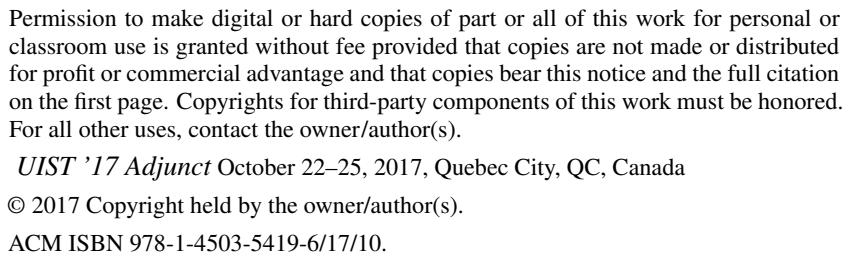
classroom use is granted without fee provided that copies are not made or distributed for profit or commercial advantage and that copies bear this notice and the full citation on the first page. Copyrights for third-party components of this work must be honored. For all other uses, contact the owner/author(s).

UIST '17 Adjunct October 22-25, 2017, Quebec City, QC, Canada

(C) 2017 Copyright held by the owner/author(s).

ACM ISBN 978-1-4503-5419-6/17/10.

DOI: https://doi .org/10.1145/3131785. 3131800 enable direct manipulation [4]. This solution restore the benefits, for example studies show that tactile feedback increases typing speed on a virtual keyboard [5].

The problem is that efficient feedback requires precise actuators, which have the same issues than physical buttons: they are expensive, are complex to manufacture, and they are fragile. Piezo actuators is a solution, because it creates precise vibrations with limited hardware [8]. However consumer electronics piezo discs are flat, and usually optimized for frequencies in the audio range.

In this demonstration, we present printed actuators and sensors. Actuators are printed with a piezo ink, sensors with a silver ink, both on a flexible substrate. These printed sensors and actuators are the basic essential components for the design of vibrotactile widgets on curved surfaces (Figure 1).

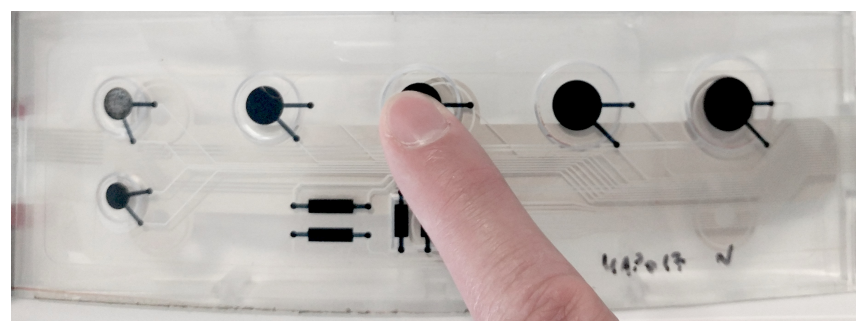

Figure 1: Printed vibrotactile buttons on a curve plastic surface

We describe the hardware design of printed actuators and sensors, and software design of interaction techniques. Then we present demonstration applications.

\section{DESIGN OF VIBROTACTILE WIDGETS}

We describe the design of vibrotactile widgets. They use printed actuators and sensors on a flexible substrate so that they can be used on curved surfaces, such as car dashboards, computer input devices, or even mobile devices. The actuators and sensors are lightweight, thin, and even transparent to a certain degree depending on a trade-off explained below.

\section{Printed actuators and sensors}

Printed actuators are unimorph : the piezoelectric EAP layer expands when the electrical field is applied, resulting in the bending of the actuator. We create vibrations with variations of the signal. The design of the actuator is a trade-off between several parameters and objectives [9]. 
The main objective is to have a sufficient movement amplitude so that the user can feel the vibration. The secondary objectives is to have transparent actuators, so that it can be stacked over a screen or backlight, and minimize power consumption. There are two ways to get higher amplitudes. The first is to use a higher voltage. This is however not possible in every application. The second is to stack several layers of actuators. The limitation is that transparency decreases as the number of layer increases. A workaround for transparency is ring actuators, or placing actuators on the sides.

The piezo ink is an Electroactive Polymer (EAP), specifically vinylidene fluoride-trifluoroethylene copolymers P(VDFTrFE) [10]. The flexible substrate is made of polyethylene naphthalate (PEN).

In order to vibrate efficiently, we clamp the actuator to ground (Figure 2). It allows the actuator to resonate similarly to a drumhead on a drum shell. The size of the free area inside the clamping influences the resonance frequency. Clamping techniques include gluing, or mechanical compression. The capacitive sensor is glued under the actuator.

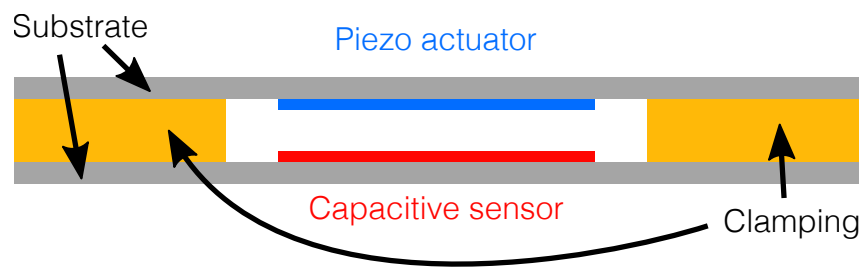

Figure 2: Cross-section of the apparatus. The top layer vibrates, and the bottom layer senses touches.

\section{Driving electronics}

Piezo actuators require a driver chip and an external power supply. We use the TI DRV2667 chip, either on the TI evaluation board, or the Fyber Labs Piezo Haptic Flex Module. We use a 5V/2A power supply, which is sufficient to drive the actuators. Capacitive sensing uses the Microchip CAP1188 driver. The capacitance values allow us to detect several levels of finger pressure on the surface. This is an essential part of the interaction techniques below. Both drivers communicate with the host computer (Raspberry Pi 3) with an I2C bus. We use a sound synthesis method for prototyping the signal [2, 6, 1], and embedded waveforms for the production setup.

\section{Interaction techniques}

The combination of precise actuators and capacitive sensors with a reasonable input range makes it possible to create vibrotactile widgets.

We designed tactile buttons by replicating Kim and Lee's method [7]. They define force-displacement curves with two types of sections: slopes and jumps, which are delimited by tactile points (Figure 4). Slopes give the user the sensation of material resistance. The resistance of the surface creates passive force feedback. In addition to that, slopes $\left(1^{\prime} \rightarrow 2\right)$ and $(2 \rightarrow 3)$ have friction grains (e.g. 20 grains per mm, each rendered with a $150 \mathrm{~Hz}$ sinusoidal wave with a release envelope of $18 \mathrm{~ms})$. Tactile points $(1,2,3)$ reproduce the click-like

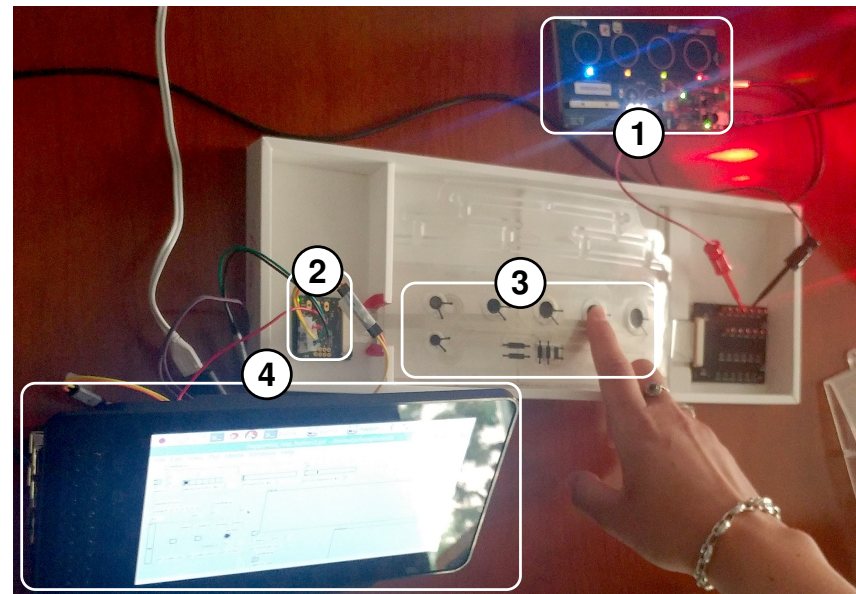

Figure 3: System with annotated components: (1) piezo driver DRV2667 demoboard, (2) capacitive sensor driver CAP1188, (3) printed actuators, (4) host computer Raspberry Pi.

sensations and are rendered as bursts of sinusoidal wave of higher frequencies, aligned with resonant frequencies of the device. (1) and (3) represent click sensation when pressing and releasing the button. (2) represents the end of the button.

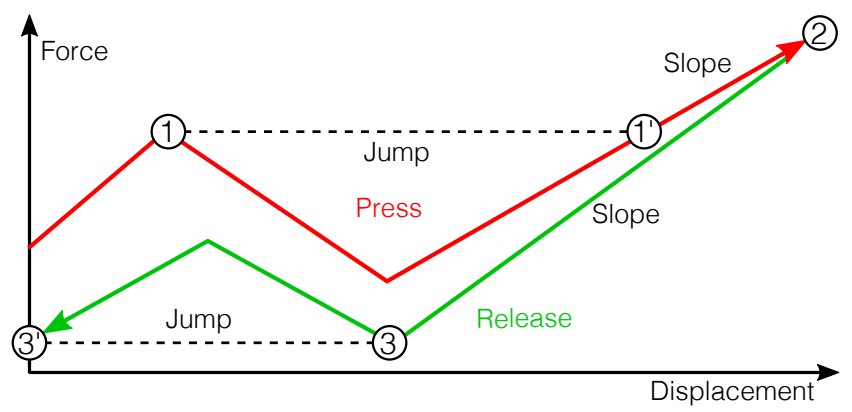

Figure 4: Force-displacement curve of a pushbutton

We extend the design of buttons towards tactile sliders, where the vibration area is larger. Therefore, the slider uses several actuators which can vibrate together or independently. We propose two designs: (1) with vibrators clamped individually, the user can feel the clamping area between actuators and interpret them as detents; (2) with all the vibrators surrounded by a large clamping area, we create detents by software depending on the finger position.

In our future work, we would like to extend the design of sliders to touchpads and multi-touch interaction; and enable tactile direct manipulation [4].

\section{ACKNOWLEDGMENTS}

We thank our partners from the HAPPINESS project, funded from European Union's Horizon 2020 research and innovation programme under grant agreement $\mathrm{N}^{\circ} 645145$. We thank Sunjun Kim and Geehyuk Lee for having helped us replicate their research by sending us a subset of their software for vibrotactile feedback generation [7]. We thank Jeff Avery from the University of Waterloo for the voice over in our demo video. 


\section{REFERENCES}

1. Ivica Ico Bukvic, Jonathan Wilkes, and Albert Gräf. 2016. Latest developments with Pd-L2Ork and its Development Branch Purr-Data. In Proceedings of the 5th International Pure Data Convention (PdCon16). 9.

2. Christian Frisson, Thomas Pietrzak, Siyan Zhao, and Ali Israr. 2016. WebAudioHaptics: Tutorial on Haptics with Web Audio. In 2nd Web Audio Conference (WAC'16). 1.

3. Masaaki Fukumoto and Toshiaki Sugimura. 2001. Active Click: Tactile Feedback for Touch Panels. In CHI 'OI Extended Abstracts on Human Factors in Computing Systems (CHI EA '01). ACM, New York, NY, USA, 121-122. DOI : http://dx.doi.org/10.1145/634067.634141

4. Aakar Gupta, Thomas Pietrzak, Nicolas Roussel, and Ravin Balakrishnan. 2016. Direct Manipulation in Tactile Displays. In Proceedings of the 2016 CHI Conference on Human Factors in Computing Systems (CHI '16). ACM, New York, NY, USA, 3683-3693. DOI :

http://dx.doi.org/10.1145/2858036.2858161

5. Eve Hoggan, Stephen A. Brewster, and Jody Johnston. 2008. Investigating the Effectiveness of Tactile Feedback for Mobile Touchscreens. In Proceedings of the SIGCHI Conference on Human Factors in Computing Systems (CHI '08). ACM, New York, NY, USA, 1573-1582. DOI : http://dx.doi.org/10.1145/1357054.1357300

6. Ali Israr, Siyan Zhao, Kyna McIntosh, Zachary Schwemler, Adam Fritz, John Mars, Job Bedford, Christian Frisson, Ivan Huerta, Maggie Kosek, Babis Koniaris, and Kenny Mitchell. 2016. Stereohaptics: A Haptic Interaction Toolkit for Tangible Virtual Experiences. In ACM SIGGRAPH 2016 Studio (SIGGRAPH'16). ACM, 13:1-13:57. DOI : http://dx.doi.org/10.1145/2929484.2970273
7. Sunjun Kim and Geehyuk Lee. 2013. Haptic Feedback Design for a Virtual Button Along Force-displacement Curves. In Proceedings of the 26th Annual ACM Symposium on User Interface Software and Technology (UIST '13). ACM, New York, NY, USA, 91-96. DOI : http://dx.doi.org/10.1145/2501988.2502041

8. Jani Lylykangas, Veikko Surakka, Katri Salminen, Jukka Raisamo, Pauli Laitinen, Kasper Rönning, and Roope Raisamo. 2011. Designing Tactile Feedback for Piezo Buttons. In Proceedings of the SIGCHI Conference on Human Factors in Computing Systems (CHI '11). ACM, New York, NY, USA, 3281-3284. DOI : http://dx.doi.org/10.1145/1978942.1979428

9. Pauline Poncet, Fabrice Casset, Antoine Latour, Fabrice Domingues Dos Santos, Sébastien Pawlak, Romain Gwoziecki, Arnaud Devos, Patrick Emery, and Stéphane Fanget. 2017. Static and Dynamic Studies of Electro-Active Polymer Actuators and Integration in a Demonstrator. Actuators 6, 2 (2017). DOI: http://dx.doi.org/10.3390/act6020018

10. Pauline Poncet, Fabrice Casset, Antoine Latour, Fabrice Domingues Dos Santos, Sébastien Pawlak, Romain Gwoziecki, and Stéphane Fanget. 2016. Design and realization of electroactive polymer actuators for transparent and flexible haptic feedback interfaces. In Proc. EuroSimE 2016. 1-5. DOI:

http://dx.doi.org/10.1109/EuroSimE. 2016.7463310 